\title{
Phase II study of the c-MET inhibitor tivantinib (ARQ 197) in patients with relapsed or relapsed/refractory multiple myeloma
}

\author{
Muhamed Baljevic $^{1,2}$ (D) - Shadia Zaman ${ }^{3}$ - Veerabhadran Baladandayuthapani ${ }^{4}$. \\ Yan Heather Lin ${ }^{4}$. Claudia Morales de Partovi ${ }^{5}$ Zuzana Berkova ${ }^{5} \cdot$ Behrang Amini $^{6}$. \\ Sheeba K. Thomas ${ }^{5}$ Jatin J. Shah ${ }^{5}$ - Donna M. Weber ${ }^{5} \cdot$ Min Fu $^{2}$. \\ Charles S. Cleeland $^{7} \cdot$ Xin Shelley Wang ${ }^{7} \cdot$ Christine M. Stellrecht $^{3} \cdot$ Richard E. Davis $^{5}$. \\ Varsha Gandhi ${ }^{3} \cdot$ Robert Z. Orlowski ${ }^{3,5}$
}

Received: 27 July 2016 / Accepted: 14 March 2017 / Published online: 23 March 2017

(C) The Author(s) 2017. This article is published with open access at Springerlink.com

\begin{abstract}
The hepatocyte growth factor/c-MET pathway has been implicated in the pathobiology of multiple myeloma, and c-MET inhibitors induce myeloma cell apoptosis, suggesting that they could be useful clinically. We conducted a phase II study with the c-MET inhibitor tivantinib in patients with relapsed, or relapsed and refractory myeloma whose disease had progressed after one to four prior therapies. Tivantinib, $360 \mathrm{mg}$ orally per dose, was administered twice daily continuously over a 4-week treatment cycle without a cap on the number of allowed cycles, barring undue toxicities or disease progression. Primary objectives were to determine the overall response rate and the toxicities of tivantinib in this patient population. Sixteen patients were enrolled in a two-stage design. Notable grade 3 and 4 hematological adverse events were limited to neutropenia in five and four patients, respec-
\end{abstract}

Muhamed Baljevic and Shadia Zaman contributed equally as first authors.

Varsha Gandhi and Robert Z. Orlowski contributed equally as senior authors.

Electronic supplementary material The online version of this article (doi:10.1007/s00277-017-2980-3) contains supplementary material, which is available to authorized users.

Robert Z. Orlowski

rorlowski@mdanderson.org

1 Division of Hematology \& Oncology, The University of Nebraska Medical Center, Omaha, NE, USA

2 Division of Cancer Medicine, The University of Texas MD Anderson Cancer Center, Houston, TX, USA

3 Department of Experimental Therapeutics, The University of Texas MD Anderson Cancer Center, Houston, TX, USA tively. Nonhematological adverse events of grade 3 or higher included hypertension (in four patients); syncope, infection, and pain (two each); and fatigue, cough, and pulmonary embolism (one each). Four of 11 evaluable patients (36\%) had stable disease as their best response, while the remainder showed disease progression. Overall, tivantinib as a single agent did not show promise for unselected relapsed/ refractory myeloma patients. However, the ability to achieve stable disease does suggest that combination regimens incorporating targeted inhibitors in patients with c-MET pathway activation could be of interest.

Keywords ARQ $197 \cdot$ Tivantinib $\cdot$ c-MET $\cdot$ Multiple myeloma $\cdot$ Relapsed
4 Department of Biostatistics, The University of Texas MD Anderson Cancer Center, Houston, TX, USA

5 Department of Lymphoma/Myeloma, The University of Texas MD Anderson Cancer Center, Houston, TX, USA

6 Department of Diagnostic Radiology, The University of Texas MD Anderson Cancer Center, Houston, TX, USA

7 Department of Symptom Research, The University of Texas MD Anderson Cancer Center, Houston, TX, USA 


\section{Introduction}

Multiple myeloma is the second most commonly diagnosed hematologic malignancy, and the total number of new cases may show a $60 \%$ increase between 2010 and 2030 [1], indicating that it is an increasing health care burden. Introduction over the past decade of proteasome inhibitors, immunomodulatory agents, and their combinations has improved the outcome of patients with relapsed and relapsed/refractory myeloma [2]. Nevertheless, patients still experience multiple relapses and decreasing remission durations with each additional line of therapy [2], and those with refractory disease in particular may not respond favorably to standard agents. Their use is limited by treatment-emergent toxicity, or by intrinsic or acquired resistance, underscoring the continued need for novel treatments.

Activation of the c-MET receptor tyrosine kinase induced by its ligand, hepatocyte growth factor (HGF), plays a role in myeloma pathobiology. Malignant plasma cells secrete HGF activator (HGFA), which converts HGF to its active form, and high HGF levels correlate with a poor prognosis [3, 4]. Syndecan 1 (CD138) on myeloma cells binds HGF and promotes c-MET signaling [5], while both potentiate interleukin6-induced growth and migration, correlating with shorter survival [6]. Preclinical studies showed that suppression of cMET signaling with small molecule inhibitors, including tivantinib, inhibited proliferation and induced apoptosis [7-10]. Preclinically, tivantinib-mediated cytotoxicity was observed at concentrations $<5 \mu \mathrm{M}$, a level achievable in the clinic that showed activity in solid tumors with a dose of $360 \mathrm{mg}$ twice daily [11]. Phase II single-agent tivantinib studies demonstrated efficacy in solid tumors [12], and the combination of tivantinib and erlotinib was tested in a phase III trial in nonsmall cell lung carcinoma and showed an improvement for the two-drug regimen in progression-free, though not overall survival [13]. Finally, biomarker studies demonstrated the efficacy of tivantinib in inhibiting MET receptor kinase, as well as inducing apoptosis of target tumor cells [11]. Together, these findings supported the hypothesis that suppressing HGF/c-MET signaling could be a rational strategy against relapsed/refractory myeloma, and we therefore performed a phase II clinical trial testing this possibility.

\section{Patients and methods}

\section{Patient selection}

Patients with histologically confirmed symptomatic myeloma by the International Myeloma Working Group (IMWG) criteria with 1-4 prior lines of therapy were eligible. They had to have measurable disease that had relapsed after, or was refractory to the prior regimen. Additional key inclusion criteria included: (1) Eastern Cooperative Oncology Group (ECOG) performance status of 0-2, (2) adequate bone marrow reserves [absolute neutrophil count (ANC) $\geq 1000$ cells/ $\mathrm{mm}^{3}$, hemoglobin $\geq 8 \mathrm{~g} / \mathrm{dL}$; platelets $\geq 100,000$ cells $\left./ \mathrm{mm}^{3}\right]$, and (3) adequate hepatic [total bilirubin $\leq 1.5 \times$ normal, aspartate and alanine aminotransferase $\leq 2.5 \times$ normal], renal [normal serum creatinine, or creatinine clearance $\geq 30 \mathrm{~mL} / \mathrm{min}$ ], and cardiac function [absence of New York Heart Association class II-IV congestive heart failure, uncontrolled angina, hypertension, myocardial infarction within 6 months, or grade 3-4 cardiac arrhythmia by NCI Common Terminology Criteria for Adverse Events (CTCAE), version 4.0]. Key exclusion criteria included: (1) central nervous system involvement, (2) previous treatment with another agent targeting HGF/c-MET, and (3) nonsecretory myeloma, active plasma cell leukemia, or POEMS syndrome (polyneuropathy, organomegaly, endocrinopathy, monoclonal gammopathy, and skin changes). The MD Anderson Institutional Review Board approved the trial. Informed consent was obtained from all patients for being included in the study. All procedures followed were in accordance with the ethical standards of the responsible committee on human experimentation (institutional and national) and with the Helsinki Declaration of 1975 , as revised in 2008. This study was registered with Clinicaltrials.gov (NCT01447914).

\section{Study design}

This open-label, phase II study used a Simon's Minimax 2stage design. A response rate of $\leq 10 \%$ was considered as clinically insignificant, while $30 \%$ was considered significant. When the probabilities of accepting a "bad" drug and of rejecting a "good" drug are both 0.1, the Simon Minimax 2stage design required 16 patients in the first stage. Another nine would have been enrolled had there been evidence of $\geq 1$ documented partial remission (PR) or better.

The primary objectives were to determine the overall response rate (ORR) to tivantinib and to define its toxicities. Secondary objectives were (1) to obtain preliminary evidence of response durability, (2) to correlate HGF/c-MET pathway activation in primary cells at baseline with efficacy, and (3) to correlate serum and marrow HGF, HGFA, and soluble c-MET (sc-MET) levels with HGF/c-MET pathway activation in primary cells at baseline and after treatment.

An exploratory objective was to evaluate the symptom burden of patients using the MD Anderson Symptom Inventory (MDASI) and its myeloma module (MDASI-MM). This brief, validated [14], patient-reported outcome tool measured 13 cancer-related symptoms and their impact on daily living. The symptoms assessed included pain, fatigue, nausea, disturbed sleep, distress, shortness of breath, difficulty remembering, lack of appetite, drowsiness, dry mouth, sadness, vomiting, numbness, constipation, muscle weakness, diarrhea, mouth or throat 
sores, rash, and trouble concentrating. Six additional items assessed symptom-related interference in general activity, mood, work, relation with others, enjoyment of life, and walking. MDASI scores were scored numerically from 0 to 10 (symptom absence to as bad as imaginable). Another exploratory objective evaluated patient-reported outcomes using the European Organization for Research on the Treatment of Cancer (EORTC) Quality of Life Questionnaire (QLQ) Core 30 (QLQ-C30) and the myeloma-specific module QLQ-MY20. Questions relating to overall health and quality of life in the QLQ-C30 were rated from 1 to 7 (very poor to excellent), while the remaining questions were graded qualitatively, in ascending severity, with not at all, a little, quite a bit, or very much.

\section{Drug dosing and assessments}

Tivantinib, provided through the National Cancer Institute Cancer Therapy Evaluation Program (CTEP), was administered at $360 \mathrm{mg}$ p.o. (three tablets of $120 \mathrm{mg}$ ) with meals twice daily of every 4-week cycle, and at this dose level, steady-state plasma tivantinib levels in a phase I trial were $7 \mu \mathrm{M}$ [11]. Treatment was to be continued unless patients experienced undue toxicities, disease progression, or withdrew consent. In the event of treatment-emergent grade 2 rash or hand-foot syndrome, grade 3 nonhematologic toxicities, or grade 4 hematologic adverse events (AEs), the drug was held until these returned to grade $0-1$ or the pretreatment baseline. At that point, tivantinib was restarted at a reduced dose (level-1 dose, $240 \mathrm{mg}$ twice daily; level-2 dose, $120 \mathrm{mg}$ twice daily).

\section{Response and toxicity assessment}

An evaluation of disease response by IMWG criteria to determine eligibility to continue therapy was to be performed after the first 2 cycles, and on day 1 of every cycle afterward. Toxicity was monitored throughout treatment, with a special focus on AEs during cycle 1. The toxicity endpoint was defined as any treatment-related unmanageable toxicity, including grade 3 nonhematologic or grade 4 hematologic effects that required delay or treatment termination during cycle 1 . Rates of these toxicities $\geq 25 \%$ were considered unacceptable.

\section{Sample collection}

Pretreatment bone marrow samples were sorted into CD $138^{+}$ and $\mathrm{CD} 138^{-}$fractions at the MD Anderson Myeloma Tissue Core Facility as described previously [15], and extracted and labeled for gene expression profiling (GEP) [16]. Plasma samples were collected on day 1 of each cycle, and isolated and stored until further analysis. HGF was determined using the HGF enzyme-linked immunosorbent assay (ELISA) (Invitrogen, Camarillo, CA) and analyzed using a Bio-Tek PowerWave XS spectrophotometer (Winooski, VT).

\section{Results}

\section{Patient characteristics}

Sixteen patients enrolled between January, 2012, and April, 2013, and received a median of 3 cycles of therapy, with one patient continuing until February, 2014. All patients were evaluable for toxicity, while 11 were formally evaluable for response after completing 2 cycles. The median patient age was 66 years, and they had received a median of 1 prior line of therapy (range 1-3). Additional demographic data are summarized in Table 1.

\section{Adverse events and treatment efficacy}

There were no unexpected toxicities given the known $\mathrm{AE}$ profile of tivantinib and no deaths. The most common AEs in $\geq 25 \%$ of patients, and felt to be at least possibly drug-related, included fatigue or decreased neutrophils ( $94 \%$ each), pain $(81 \%)$, myalgias (56\%), diarrhea (38\%), memory impairment, respiratory disorders, and rash (31\% each), hypertension $(25 \%)$, and these were predominantly grades $1-2$ (Table 2 ). Grade 3 or 4 AEs included neutropenia (31 and 25\%, respectively), syncope, infection, pain (13\% of each, all grade 3), and anal fissure, cough, fatigue, hypertension, and pulmonary embolism (6\% each, all grade 3 ).

Stable disease (SD) was observed as the best response in 4/ $11(36 \%)$ evaluable patients, or $4 / 16(25 \%)$ patients who were enrolled and received at least one dose of drug. This was maintained for up to 15 cycles in patient 12 , who withdrew consent because other therapies were available, while the remaining 7/11 (63\%) patients showed progression (PD). Among the five inevaluable patients, treatment was stopped prior to completing 2 cycles because of toxicity in two patients (syncope/bradycardia and neutropenic fever), withdrawal of consent in one, and PD in two patients (both showing a progressing Bence-Jones paraprotein). While the protocol did allow replacement of patients who were inevaluable for response, after consultation with CTEP, and given the lack of activity, a decision was made to halt enrollment.

Prior therapies received by the patients who experienced SD included thalidomide and dexamethasone (Td) leading to autologous stem cell transplantation (ASCT), and then bortezomib and dexamethasone $(\mathrm{Vd})$ at first relapse in patient $2 ; \mathrm{Vd}$, followed by bortezomib and pegylated liposomal doxorubicin after first progression in patient $4 ; \mathrm{Td}$ and ASCT in patient 5 ; and $\mathrm{Vd}$, followed after progression by lenalidomide, bortezomib, and dexamethasone and then ASCT in patient 12. The monoclonal protein (M-protein) evolution prior to, and after, initiation of tivantinib therapy in SD patients who received more than 2 cycles of therapy is depicted in Fig. 1. All three patients had rising M-proteins at enrollment (patient 12 also had progressing Bence-Jones paraprotein, while patient 5 
Table 1 Baseline demographic and clinical characteristics of patients enrolled in this phase II study of tivantinib $(N=16)$

\begin{tabular}{|c|c|c|}
\hline Characteristic & No. & $\%$ \\
\hline Age (median, range) & \multicolumn{2}{|c|}{$66.3(49-76)$} \\
\hline$\leq 65$ years & 6 & 37.5 \\
\hline$>65$ years & 10 & 62.5 \\
\hline \multicolumn{3}{|l|}{ Sex } \\
\hline Male & 9 & 56.25 \\
\hline Female & 7 & 43.75 \\
\hline \multicolumn{3}{|l|}{ Race } \\
\hline African-American & 2 & 12.5 \\
\hline Asian & 1 & 6.25 \\
\hline Caucasian & 13 & 81.25 \\
\hline \multicolumn{3}{|l|}{ Ethnicity } \\
\hline Hispanic & 3 & 18.75 \\
\hline Non-Hispanic & 13 & 81.25 \\
\hline \multicolumn{3}{|l|}{ Disease status } \\
\hline Relapsed & 13 & 81.25 \\
\hline Refractory & 3 & 18.75 \\
\hline \multicolumn{3}{|l|}{ Prior autologous stem cell transplantation } \\
\hline Yes & 10 & 62.5 \\
\hline No & 6 & 37.5 \\
\hline Prior lines of therapy (median, range) & \multicolumn{2}{|c|}{$2.5(2-3)$} \\
\hline \multicolumn{3}{|l|}{ Types of prior therapies } \\
\hline Bortezomib/lenalidomide/dexamethasone & 6 & 37.5 \\
\hline Cyclophosphamide/bortezomib/dexamethasone & 4 & 25.0 \\
\hline Vincristine/doxorubicin/dexamethasone & 1 & 6.25 \\
\hline Bortezomib/lenalidomide & 1 & 6.25 \\
\hline Bortezomib/Liposomal doxorubicin & 1 & 6.25 \\
\hline Bortezomib/dexamethasone & 5 & 31.25 \\
\hline Thalidomide/dexamethasone & 3 & 18.75 \\
\hline Lenalidomide/dexamethasone & 5 & 31.25 \\
\hline Bortezomib & 2 & 12.5 \\
\hline Lenalidomide & 3 & 18.75 \\
\hline Dexamethasone & 1 & 6.25 \\
\hline
\end{tabular}

had progressing serum free light chains and worsening thrombocytopenia) and met criteria for SD, though further exposure to tivantinib yielded evidence of some benefit only in patient 12. In patients with $\mathrm{SD}$, the median durability of response was 7 cycles (range $2-15$ ) or 6.5 months (range $2-15$ ). Two SD patients ultimately withdrew consent (patients 2 and 5), while patient 4 developed declining performance status that led to discontinuation of treatment.

\section{Symptom burden and quality of life}

A total of 95 MDASI and 93 EORTC measurements were collected, $63 \%$ of which were within 3 cycles of therapy. The top five most severe MDASI symptoms were fatigue, pain, numbness, limited activity, and muscle weakness (Table 3). During the first cycle, five patients were asymptomatic (scoring 0-3), and four of these completed at least 3 cycles of therapy and remained asymptomatic. Two patients reported $>10$ symptoms as moderate/severe (scoring 4-10) that significantly interfered with their activity and enjoyment of life; most of those symptoms did not improve over time. Most commonly reported EORTC symptoms during cycle 1 related to tiredness, feeling less attractive, thinking about illness, weakness, and pain interference with daily activities, while the overall top five EORTC concerns were problems with strenuous activities, tiredness, pain/aches, weakness, and problem with long walks (Table 4). The QLQ-C30 questions on overall health and quality of life were scored high consistently during the treatment despite certain patients experiencing PD. Interestingly, both parameters decreased in two patients with SD.

\section{Plasma HGF levels}

A total of 79 plasma samples were collected, and two samples were collected from healthy donors. The median plasma HGF level in myeloma patients was $450 \mathrm{pg} / \mathrm{mL}$ (range 150-5991) (Fig. 2, A-C) at baseline and was $<600 \mathrm{pg} / \mathrm{mL}$ in the majority (10/16), while the HGF levels in the two healthy donors were 60 and $1400 \mathrm{pg} / \mathrm{mL}$. Only two patients with evaluable responses had HGF levels $\geq 600 \mathrm{pg} / \mathrm{mL}$. The median HGF level in four patients with SD was $337 \mathrm{pg} / \mathrm{mL}$ (150-999), which was only slightly lower than in patients who had PD, $419 \mathrm{pg} /$ $\mathrm{mL}$ (236-1447). There was no correlation between the basal level of HGF and response among patients who achieved SD versus those who did not $(P=0.783)$. Four of the five patients not evaluable for response, and one patient who had PD after 3 cycles, had high HGF levels, with a median value of $2891 \mathrm{pg} / \mathrm{mL}$ (577.7-11,976; Fig. 2a, c). Of the patients who had high HGF levels, only $20 \%$ (1 patient) stayed on treatment for $>2$ cycles, while $73 \%$ with low levels stayed on treatment for $>2$ cycles (Fig. 2a, b). Although there were no significant differences in the HGF levels between SD and PD groups at baseline, HGF levels decreased in three of four patients that achieved SD, while of the six evaluable patients with PD, HGF increased in four during therapy (Fig. 2a, b).

\section{Gene expression profiling}

Of the $12 \mathrm{CD} 138^{+}$samples available, 9 provided RNA suitable for GEP. The obtained data were analyzed by gene set enrichment analysis (GSEA) run between the high $(n=2)$ versus low HGF $(n=7)$ groups. Because the sample number was low in the high HGF group, a $t$ test could not be conducted, and the two high HGF samples available were from inevaluable patients. To identify a gene set that was enriched in the high versus low HGF group, we selected gene sets with a false discovery rate of $<0.05$. This analysis revealed that 
Table 2 Hematologic and nonhematologic toxicities in patients treated with tivantinib $(N=16)$

\begin{tabular}{|c|c|c|c|c|c|c|c|c|}
\hline \multirow[t]{2}{*}{ Toxicity grade } & \multicolumn{2}{|l|}{1} & \multicolumn{2}{|l|}{2} & \multicolumn{2}{|l|}{3} & \multicolumn{2}{|l|}{4} \\
\hline & No. & $\%$ & No. & $\%$ & No. & $\%$ & No. & $\%$ \\
\hline \multicolumn{9}{|l|}{ Hematological toxicity type } \\
\hline Neutropenia & & & 15 & 93.8 & 5 & 31.3 & 4 & 25.0 \\
\hline Anemia & & & 3 & 18.8 & & & & \\
\hline Thrombocytopenia $^{\mathrm{a}}$ & 2 & 12.5 & & & & & & \\
\hline \multicolumn{9}{|l|}{ Nonhematological toxicity type } \\
\hline Hypertension & & & & & 4 & 25.0 & & \\
\hline Pain & & & 13 & 81.3 & 2 & 12.5 & & \\
\hline Syncope & & & & & 2 & 12.5 & & \\
\hline Infection & & & & & 2 & 12.5 & & \\
\hline Fatigue & 15 & 93.8 & & & 1 & 6.3 & & \\
\hline Cough $^{\mathrm{a}}$ & 7 & 43.8 & & & 1 & 6.3 & & \\
\hline Pulmonary embolus(i) & & & & & 1 & 6.3 & & \\
\hline Sinus bradycardia & & & 3 & 18.8 & & & & \\
\hline Allergic rhinitis ${ }^{\mathrm{a}}$ & & & 2 & 12.5 & & & & \\
\hline Insomnia $^{\mathrm{a}}$ & & & 2 & 12.5 & & & & \\
\hline Metabolism/ND ${ }^{\mathrm{a}}$ & & & 2 & 12.5 & & & & \\
\hline Musculoskeletal/connective TD & & & 2 & 12.5 & & & & \\
\hline Peripheral sensory neuropathy ${ }^{\mathrm{a}}$ & 10 & 62.5 & & & & & & \\
\hline Myalgia & 9 & 56.3 & & & & & & \\
\hline Skin/subcutaneous TD ${ }^{\mathrm{a}}$ & 8 & 50.0 & & & & & & \\
\hline Diarrhea & 6 & 37.5 & & & & & & \\
\hline Constipation $^{\mathrm{a}}$ & 5 & 31.3 & & & & & & \\
\hline Renal/urinary disorder ${ }^{\mathrm{a}}$ & 5 & 31.3 & & & & & & \\
\hline Respiratory disorder & 5 & 31.3 & & & & & & \\
\hline Maculopapular rash & 5 & 31.3 & & & & & & \\
\hline Memory impairment & 5 & 31.3 & & & & & & \\
\hline Blurred vision $^{\mathrm{a}}$ & 5 & 31.3 & & & & & & \\
\hline Dry eye $\mathrm{e}^{\mathrm{a}}$ & 4 & 25.0 & & & & & & \\
\hline Limb edema ${ }^{a}$ & 3 & 18.8 & & & & & & \\
\hline Fever & 2 & 12.5 & & & & & & \\
\hline Headache & 2 & 12.5 & & & & & & \\
\hline Dizziness $^{\mathrm{a}}$ & 2 & 12.5 & & & & & & \\
\hline Gastrointestinal disorder ${ }^{\mathrm{a}}$ & 2 & 12.5 & & & & & & \\
\hline Nausea & 2 & 12.5 & & & & & & \\
\hline Watery eyes & 2 & 12.5 & & & & & & \\
\hline Alopecia & 2 & 12.5 & & & & & & \\
\hline Dry skin ${ }^{\mathrm{a}}$ & 2 & 12.5 & & & & & & \\
\hline Anxiety $^{a}$ & 2 & 12.5 & & & & & & \\
\hline
\end{tabular}

$N D$ nutritional deficiency, $T D$ tissue disorder

${ }^{a}$ Unrelated to the treatment interferon response genes were associated with the high HGF phenotype (Supplementary Table 1). One differentially expressed gene was Interferon regulatory factor (IRF) 4, which is required for production of immunoglobulinsecreting plasma cells [17]. In our GEP analysis, samples from patients who had high HGF had higher average IRF4 expression compared to samples from patients with low HGF (Supplementary Fig. 1). Moreover, two patients who had high HGF levels were on the trial for $<2$ cycles due to $\mathrm{PD}$ and were not evaluable for response. 
Fig. 1 Monoclonal protein evolution prior to and during tivantinib therapy for stable disease patients. Only patients 2 , 5 , and 12 , who experienced longer durations of therapy on protocol, are depicted

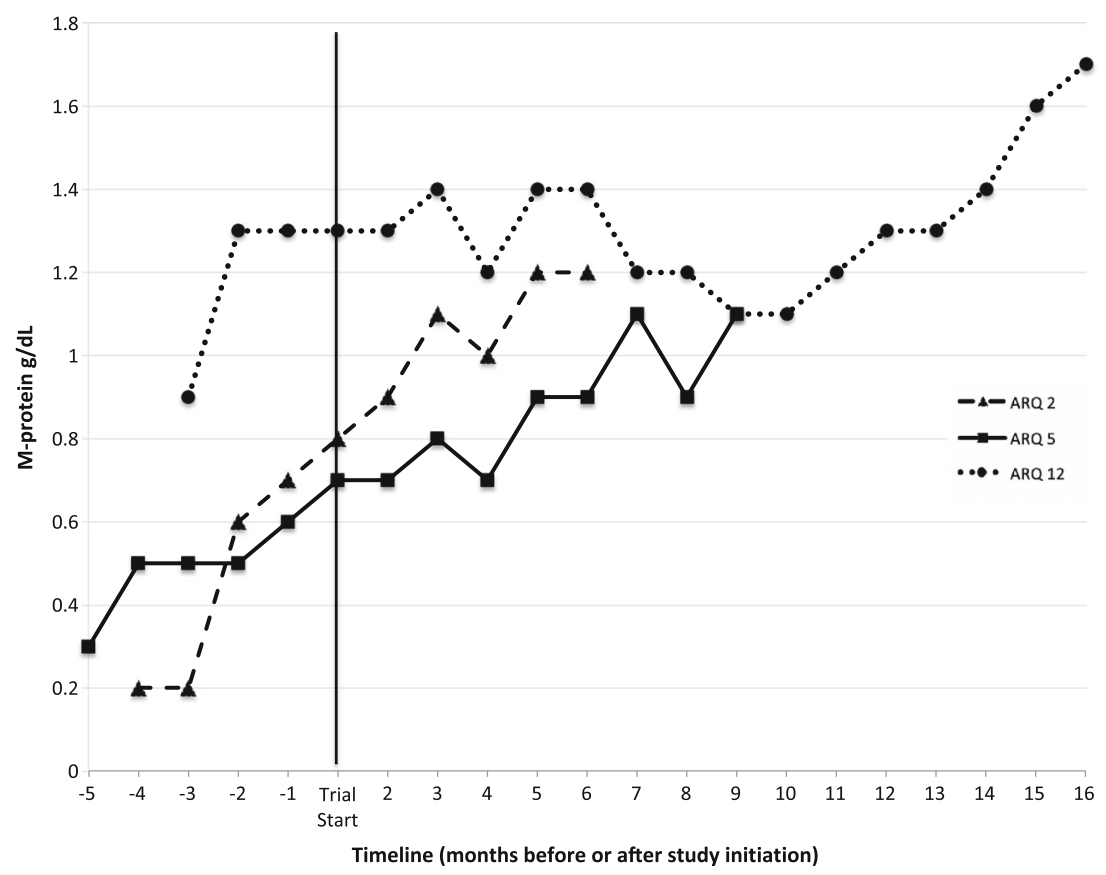

\section{Discussion}

The c-MET receptor tyrosine kinase proto-oncogene regulates cell growth, survival, and migration, and c-MET signaling after engagement of its ligand, HGF, is a contributor to the pathogenesis of myeloma. HGF and c-MET expression at the mRNA and protein levels has been found in most myeloma cell lines and primary samples $[18,19]$. Studies correlating HGF levels with clinical parameters showed that HGF was elevated at diagnosis in the serum and marrows of patients [20-22] and that HGF levels were elevated in patients with more aggressive disease [21-23], correlating with an inferior prognosis [22, 24, 25]. Conversely, declining HGF levels are seen in patients responding to anti-myeloma therapies [26-28], and patients with low HGF levels were more likely to achieve high-quality responses [26, 29]. Furthermore, reduction of MET using shRNAs [30] or a ribozyme [31] resulted in growth inhibition and apoptosis of myeloma cell lines. Collectively, these findings provided a rationale for studying tivantinib, a small-molecule, non-ATP-competitive c-MET inhibitor which binds to and stabilizes inactive c-MET [32].

Tivantinib was well-tolerated in our patients, and the toxicities were mild and manageable, with a low rate of grade 3-4 events consistent with the known AE profile of this agent.
Table 3 The most common reported MD Anderson Symptom Inventory (MDASI) symptoms and their severity during tivantinib treatment

\begin{tabular}{|c|c|c|c|c|c|}
\hline \multirow{2}{*}{$\begin{array}{l}\text { Trial } \\
\text { Patient }\end{array}$} & \multicolumn{5}{|c|}{ MDASI Symptom Score, median (range) } \\
\hline & Pain & Fatigue & Numbness & Muscle weakness & Limited activity \\
\hline ARQ 1 & $0(0)$ & $2(0-2)$ & $0(0)$ & $0(0-2)$ & $0(0)$ \\
\hline ARQ $2^{\mathrm{a}}$ & $2(0-2)$ & $3(0-7)$ & $1(1-3)$ & $0(0-2)$ & $0(0-1)$ \\
\hline ARQ 3 & $6(2-6)$ & $5(3-7)$ & $7(4-8)$ & $5(3-8)$ & $6(4-8)$ \\
\hline ARQ $4^{\mathrm{a}}$ & $0(0)$ & $0(0)$ & $0(0-7)$ & $0(0)$ & $0(0)$ \\
\hline ARQ $5^{\mathrm{a}}$ & $4.5(1-6)$ & $3(1-6)$ & $0(0)$ & $0(0)$ & $0(0-1)$ \\
\hline ARQ 6 & $6(5-7)$ & $8(6-9)$ & $7(6-8)$ & $4(4-6)$ & $5(4-7)$ \\
\hline ARQ 7 & $0(0)$ & $0(0)$ & $2(0-2)$ & $0(0)$ & $0(0)$ \\
\hline ARQ 9 & $0(0)$ & $0(0)$ & $0(0)$ & $0(0)$ & $0(0)$ \\
\hline ARQ $12^{\mathrm{a}}$ & $1(0-1)$ & $0(0)$ & $0(0)$ & $0(0)$ & $0(0)$ \\
\hline ARQ 13 & $1(1-2)$ & $1(1-2)$ & $0(0)$ & $0(0-1)$ & $1(1)$ \\
\hline ARQ 14 & $0(0)$ & $2(1-2)$ & $0(0)$ & $0(0)$ & $0.5(0-2)$ \\
\hline
\end{tabular}

${ }^{\text {a }}$ Denotes four patients who had stable disease response 
Table 4 The most commonly reported EORTC QLQ-C30 (multiple myeloma) symptoms and their severity during tivantinib treatment

Trial patient EORTC QLQ-C30 Multiple Myeloma Symptom Score

Qualitative measures (Not at all $=1^{\mathrm{a}} ;$ A little $=2^{\mathrm{a}}$; Quite a bit $=3^{\mathrm{a}}$; Very much $=4^{\mathrm{a}}$ ), median Quantitative measures $[1-7]$, median (range) (range)

\begin{tabular}{llllllll} 
& $\begin{array}{l}\text { Trouble dong } \\
\text { strenuous activities }\end{array}$ & Long walk problem & Pain/aches & Weakness & Tiredness & Overall health & Overall quality of life \\
\hline ARQ 1 & $1(1)$ & $1(1-2)$ & $1(1)$ & $1(1)$ & $1(1-2)$ & $7(6-7)$ & $7(6-7)$ \\
ARQ 2 $^{\mathrm{b}}$ & $2(1-2)$ & $2(1-3)$ & $1(1)$ & $2(1-3)$ & $1(1-3)$ & $4(4-6)$ & $5(3-6)$ \\
ARQ 3 & $3(2-4)$ & $2(1-3)$ & $3(2-3)$ & $3(2-3)$ & $3(2-3)$ & $4(3-5)$ & $4(2-5)$ \\
ARQ 4 $^{\text {b }}$ & $1(1-2)$ & $1(1)$ & $1(1)$ & $1(1)$ & $1(1-3)$ & $7(6-7)$ & $7(6-7)$ \\
ARQ 5 $^{\mathrm{b}}$ & $1(1)$ & $1(1-2)$ & $1(1-3)$ & $1(1)$ & $2(1-2)$ & $6(4-6)$ & $6(5-7)$ \\
ARQ 6 & $3(3)$ & $3(3)$ & $2(2-3)$ & $3(3-4)$ & $3(3-4)$ & $5(2-6)$ & $6(3-6)$ \\
ARQ 7 & $1(1)$ & $1(1)$ & $1(1)$ & $1(1)$ & $1(1)$ & $7(7)$ & $7(7)$ \\
ARQ 9 & $1(1-4)$ & $1(1)$ & $1(1)$ & $1(1)$ & $1(1)$ & $7(7)$ & $7(7)$ \\
ARQ 12 & $1(1-2)$ & $3(3)$ & $1(1-2)$ & $1(1)$ & $1(1-2)$ & $7(2-7)$ & $7(1-7)$ \\
ARQ 13 & $3(3)$ & $1(1)$ & $1(1)$ & $1(1)$ & $2(1-2)$ & $7(6-7)$ & $7(7)$ \\
ARQ 14 & $1(1)$ & & $1(1)$ & $2(2)$ & $6(6)$ & $7(7)$ \\
\hline
\end{tabular}

EORTC QLQ-C30 European Organization for Research and Treatment of Cancer Quality of Life Questionnaire C30

${ }^{a}$ Qualitative measures assigned numbers to allow quantification of symptoms

${ }^{\mathrm{b}}$ Denotes four patients who had stable disease response

This conclusion is supported by data collected using the MDASI, which did not indicate that therapy was poorly tolerated, with only few patients indicating $>10$ symptoms affecting their activity and the enjoyment of life negatively on treatment. Quality of life measurements using the EORTC QLQ-C30 and QLQ-MY20 were similarly indicative of relatively good tolerance, with overall health and quality of life judged as great by the majority.

Stable disease was seen in 4/11 evaluable patients as the best response, yielding a disease control rate of $36 \%$, but no patients had a PR or better. A recent study by Lendvai et al. [33] evaluated the activity of cabozantinib, a multi-targeted tyrosine kinase inhibitor that suppresses MET activation. Among 12 patients, one minimal response was seen, eight patients had SD, and two suffered PD. One explanation for this lack of activity could be that HGF levels, while variable among our patients, were mostly in the low range and were not reported by Lendvai and colleagues. Of note, clinical standards for HGF levels have not been established, and we therefore based our cutoff for high vs. low based on data from the literature and from the data we obtained by examining the HGF levels in the two healthy donors (Fig. 2c). Data from Verstovsek et al. determined the HGF level in normal plasma samples to be $164-522.3 \mathrm{pg} / \mathrm{mL}$, while the data from Sakon
A

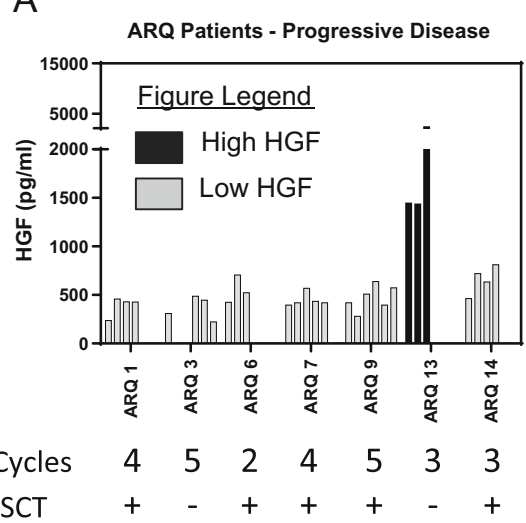

B

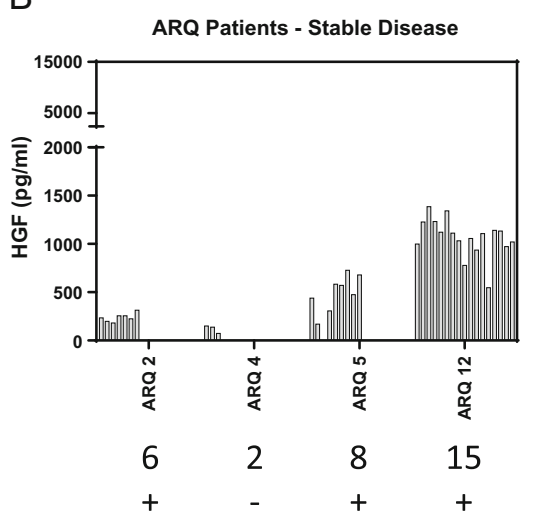

C

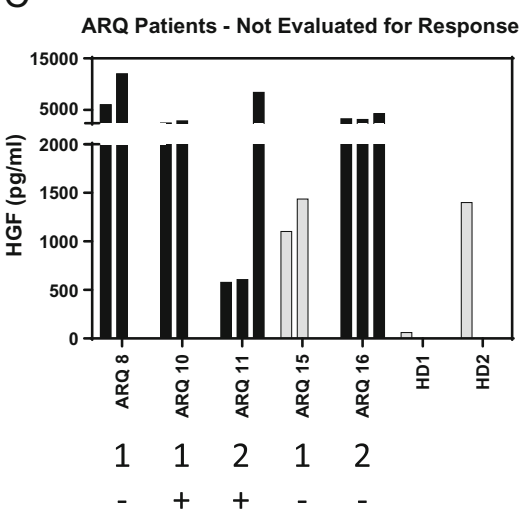

Fig. 2 Plasma HGF levels in evaluable patients. Plasma HGF levels $(\mathrm{pg} / \mathrm{mL})$ were measured by ELISA in patients with a progressive disease, $\mathbf{b}$ stable disease, and $\mathbf{c}$ patients who were not evaluable for response. The number of cycles of tivantinib received, and whether the patient had prior autologous stem cell transplant (Auto SCT), is indicated. High HGF samples are labeled with a black bar, and low HGF samples are labeled with a gray bar. HD indicates healthy donors used as controls 
et al. determined the HGF level in normal plasma samples to be $90 \mathrm{pg} / \mathrm{mL}$. Our analysis of HGF levels in two healthy donors yielded levels of 60 and $1400 \mathrm{pg} / \mathrm{mL}$. Therefore, we conservatively set the high HGF level to be any value $>2000 \mathrm{pg} / \mathrm{mL}$, and low to be $\leq 2000 \mathrm{pg} / \mathrm{mL}[34,35]$. If we accept these definitions, this suggests the possibility that the myeloma cells from our patients were not especially dependent on $\mathrm{HGF} / \mathrm{c}-\mathrm{MET}$ signaling and that selected patients with high HGF levels could have fared better. While speculative, this hypothesis is supported by the observation that HGF levels generally tended to decrease for patients with SD compared to those who progressed. Furthermore, two patients who had high HGF levels and high IRF4 expression were on the trial for only 1 cycle each due to PD, suggesting a worse disease biology. However, median HGF levels between the two groups did not differ statistically, and the small sample size limits the ability to make strong conclusions.

After completion of our patient enrollment, several groups published data questioning the role of tivantinib as a specific MET inhibitor. Basilico et al. noted that tivantinib exerted cytotoxic activity that was not influenced by c-MET gene copy number [36]. Katayama et al. found that tivantinib showed similar potency against c-MET-addicted and nonaddicted cells [37] and that tivantinib induced a $\mathrm{G}_{2} / \mathrm{M}$ arrest, while other c-MET inhibitors induced $G_{0} / G_{1}$ arrest. Finally, in silico studies identified microtubules as potential tivantinib targets, and microtubule disruption was noted in treated cell lines. This led Katayama and colleagues to conclude that tivantinib inhibited microtubule polymerization in addition to inhibiting c-MET. Perhaps because the growth fraction of human myeloma cells is typically low, microtubule-targeting agents have not shown strong activity in this disease. If tivantinib does indeed work in part as a microtubule inhibitor, this could also explain its lack of efficacy in our patients.

Given the questions surrounding tivantinib, this study and the trial of Lendvai et al. with cabozantinib have not eliminated the possibility that targeting c-MET may be a useful treatment strategy for some relapsed and/or refractory myeloma patients. It would be rational to consider using more specific inhibitors in patients with high HGF levels or, better yet, with c-MET pathway activation judged by GEP or phospho-cMET levels. Further, combination strategies with other antimyeloma therapeutics, including proteasome inhibitors, may be an attractive option, since c-MET activation may be associated with clinical drug resistance [38].

\footnotetext{
Acknowledgements We thank the patients and their families for participating in this study. This work was supported by funding from the National Cancer Institute (NCI) to MD Anderson Cancer Center in the form of the SPORE in Multiple Myeloma (P50 CA142509), the Cancer Center Support Grant (P30 CA16672), and CTEP grant N01 CM-20110039. V.G. would like to acknowledge a Leukemia and Leukemia Society Translational Research Program award and the Multiple Myeloma
}

SPORE. R.Z.O., the Florence Maude Thomas Cancer Research Professor, acknowledges support from the NCI (U10 CA032102, R01 CA184464, CA194264), the MD Anderson Cancer Center High-Risk Multiple Myeloma Moon Shot, and thanks the Brock Family Myeloma Research Fund, the Yates Ortiz Myeloma Fund, and the Diane \& John Grace Family Foundation.

Authorship contributions M.B. analyzed data and wrote the manuscript. S.Z. designed and conducted correlative studies and edited the manuscript. V.B. and Y.H.L. designed the trial and analyzed data. C.M.P. consented patients and collected clinical data. Z.B. and B.A. analyzed data and wrote the manuscript. S.K.T., J.J.S., D.M.W., and M.W. treated patients and reviewed/edited the manuscript. M.F. performed correlative analysis. C.C.S. and X.S.W. collected and analyzed patient data. C.M.S. and. V.G. participated in the protocol design, analyzed data, and reviewed the manuscript. R.Z.O. designed the trial, treated patients, analyzed data, and helped write and edit the manuscript.

\section{Compliance with ethical standards}

Conflict of interest The authors declare no conflicts of interest relevant to this study.

Open Access This article is distributed under the terms of the Creative Commons Attribution 4.0 International License (http:// creativecommons.org/licenses/by/4.0/), which permits unrestricted use, distribution, and reproduction in any medium, provided you give appropriate credit to the original author(s) and the source, provide a link to the Creative Commons license, and indicate if changes were made.

\section{References}

1. Smith BD, Smith GL, Hurria A, Hortobagyi GN, Buchholz TA (2009) Future of cancer incidence in the United States: burdens upon an aging, changing nation. J Clin Oncol Off J Am Soc Clin Oncol 27:2758-2765

2. Kumar SK, Lee JH, Lahuerta JJ et al (2012) Risk of progression and survival in multiple myeloma relapsing after therapy with IMiDs and bortezomib: a multicenter international myeloma working group study. Leukemia 26:149-157

3. Tjin EPM, Derksen PWB, Kataoka H, Spaargaren M, Pals ST (2004) Multiple myeloma cells catalyze hepatocyte growth factor (HGF) activation by secreting the serine protease HGF-activator. Blood 104:2172-2175

4. Wader KF, Fagerli UM, Holt RU et al (2008) Elevated serum concentrations of activated hepatocyte growth factor activator in patients with multiple myeloma. Eur J Haematol 81:380-383

5. Derksen PWB, Keehnen RMJ, Evers LM, van Oers MHJ, Spaargaren M, Pals ST (2002) Cell surface proteoglycan syndecan-1 mediates hepatocyte growth factor binding and promotes met signaling in multiple myeloma. Blood 99:1405-1410

6. Andersen NF, Standal T, Nielsen JL et al (2005) Syndecan-1 and angiogenic cytokines in multiple myeloma: correlation with bone marrow angiogenesis and survival. Br J Haematol 128:210-217

7. Hov H, Holt RU, Rø TB et al (2004) A selective c-met inhibitor blocks an autocrine hepatocyte growth factor growth loop in ANBL-6 cells and prevents migration and adhesion of myeloma cells. Clinical cancer research : an official journal of the American Association for Cancer Research 10:6686-6694

8. Phillip CJ, Zaman S, Shentu S et al (2013) Targeting MET kinase with the small-molecule inhibitor amuvatinib induces cytotoxicity in primary myeloma cells and cell lines. J Hematol Oncol 6:92 
9. Ferrucci A, Moschetta M, Frassanito MA et al (2014) A HGF/ cMET autocrine loop is operative in multiple myeloma bone marrow endothelial cells and may represent a novel therapeutic target. Clinical cancer research: an official journal of the American Association for Cancer Research. 20:5796-5807

10. Zaman S, Shentu S, Yang J et al (2015) Targeting the pro-survival protein MET with tivantinib (ARQ 197) inhibits growth of multiple myeloma cells. Neoplasia 17:289-300

11. Yap TA, Olmos D, Brunetto AT et al (2011) Phase I trial of a selective c-MET inhibitor ARQ 197 incorporating proof of mechanism pharmacodynamic studies. J Clin Oncol Off J Am Soc Clin Oncol 29:1271-1279

12. Wagner AJ, Goldberg JM, Dubois SG et al (2012) Tivantinib (ARQ 197), a selective inhibitor of MET, in patients with microphthalmia transcription factor-associated tumors: results of a multicenter phase 2 trial. Cancer 118:5894-5902

13. Scagliotti G, von Pawel J, Novello S et al (2015) Phase III multinational, randomized, double-blind, placebo-controlled study of Tivantinib (ARQ 197) plus Erlotinib versus Erlotinib alone in previously treated patients with locally advanced or metastatic Nonsquamous non-small-cell lung cancer. J Clin Oncol Off J Am Soc Clin Oncol 33:2667-2674

14. Jones D, Vichaya EG, Wang XS et al (2013) Validation of the M. D. Anderson symptom Inventory multiple myeloma module. J Hematol Oncol 6:13

15. Bjorklund CC, Ma W, Wang Z-Q et al (2011) Evidence of a role for activation of Wnt/beta-catenin signaling in the resistance of plasma cells to lenalidomide. J Biol Chem 286:11009-11020

16. Ma W, Wang M, Wang ZQ et al (2010) Effect of long-term storage in TRIzol on microarray-based gene expression profiling. Cancer Epidemiol Biomark Prev 19:2445-2452

17. Shaffer AL, Emre NC, Lamy L et al (2008) IRF4 addiction in multiple myeloma. Nature 454:226-231

18. Börset M, Hjorth-Hansen H, Seidel C, Sundan A, Waage A (1996) Hepatocyte growth factor and its receptor c-met in multiple myeloma. Blood 88:3998-4004

19. Borset M, Lien E, Espevik T, Helseth E, Waage A, Sundan A (1996) Concomitant expression of hepatocyte growth factor/ scatter factor and the receptor c-MET in human myeloma cell lines. J Biol Chem 271:24655-24661

20. Seidel C, Børset M, Turesson I, Abildgaard N, Sundan A, Waage A (1998) Elevated serum concentrations of hepatocyte growth factor in patients with multiple myeloma. The Nordic myeloma study group. Blood 91:806-812

21. Iwasaki T, Hamano T, Ogata A, Hashimoto N, Kitano M, Kakishita E (2002) Clinical significance of vascular endothelial growth factor and hepatocyte growth factor in multiple myeloma. $\mathrm{Br} \mathrm{J}$ Haematol 116:796-802

22. Seidel C, Lenhoff S, Brabrand S et al (2002) Hepatocyte growth factor in myeloma patients treated with high-dose chemotherapy. $\mathrm{Br}$ J Haematol 119:672-676

23. Alexandrakis MG, Passam FH, Sfiridaki A, Kandidaki E, Roussou P, Kyriakou DS (2003) Elevated serum concentration of hepatocyte growth factor in patients with multiple myeloma: correlation with markers of disease activity. Am J Hematol 72:229-233
24. Kara IO, Sahin B, Gunesacar R, Unsal C (2006) Clinical significance of hepatocyte growth factor, platelet-derived growth factor$\mathrm{AB}$, and transforming growth factor-alpha in bone marrow and peripheral blood of patients with multiple myeloma. Adv Ther 23: 635-645

25. Turesson I, Abildgaard N, Ahlgren T et al (1999) Prognostic evaluation in multiple myeloma: an analysis of the impact of new prognostic factors. Br J Haematol 106:1005-1012

26. Ludek P, Hana S, Zdenek A et al (2010) Treatment response to bortezomib in multiple myeloma correlates with plasma hepatocyte growth factor concentration and bone marrow thrombospondin concentration. Eur J Haematol 84:332-336

27. Sezer O, Jakob C, Eucker J et al (2001) Serum levels of the angiogenic cytokines basic fibroblast growth factor (bFGF), vascular endothelial growth factor (VEGF) and hepatocyte growth factor (HGF) in multiple myeloma. Eur J Haematol 66:83-88

28. Pour L, Svachova H, Adam Z et al (2010a) Levels of angiogenic factors in patients with multiple myeloma correlate with treatment response. Ann Hematol 89:385-389

29. Pour L, Svachova H, Adam Z et al (2010b) Pretreatment hepatocyte growth factor and thrombospondin-1 levels predict response to high-dose chemotherapy for multiple myeloma. Neoplasma 57: 29-34

30. Phillip CJ, Stellrecht CM, Nimmanapalli R, Gandhi V (2009) Targeting MET transcription as a therapeutic strategy in multiple myeloma. Cancer Chemother Pharmacol 63:587-597

31. Stellrecht CM, Phillip CJ, Cervantes-Gomez F, Gandhi V (2007) Multiple myeloma cell killing by depletion of the MET receptor tyrosine kinase. Cancer Res 67:9913-9920

32. Eathiraj S, Palma R, Volckova E et al (2011) Discovery of a novel mode of protein kinase inhibition characterized by the mechanism of inhibition of human mesenchymal-epithelial transition factor (cmet) protein autophosphorylation by ARQ 197. J Biol Chem 286: 20666-20676

33. Lendvai N, Yee AJ, Tsakos I et al (2016) Phase IB study of cabozantinib in patients with relapsed and/or refractory multiple myeloma. Blood 127(19):2355-2356

34. Verstovsek S, Kantarjian H, Estey E et al (2001) Plasma hepatocyte growth factor is a prognostic factor in patients with acute myeloid leukemia but not in patients with myelodysplastic syndrome. Leukemia 15:1165-1170

35. Sakon M, Kita Y, Takeda Y et al (1999) Measurement of hepatocyte growth factor in serum and plasma. Int J Clin Lab Res 29:110-113

36. Basilico C, Pennacchietti S, Vigna E et al (2013) Tivantinib (ARQ197) displays cytotoxic activity that is independent of its ability to bind MET. Clinical cancer research: an official journal of the American Association for Cancer Research. 19:2381-2392

37. Katayama R, Aoyama A, Yamori T et al (2013) Cytotoxic activity of tivantinib (ARQ 197) is not due solely to c-MET inhibition. Cancer Res 73:3087-3096

38. Moschetta M, Basile A, Ferrucci A et al (2013) Novel targeting of phospho-cMET overcomes drug resistance and induces antitumor activity in multiple myeloma. Clinical cancer research: an official journal of the American Association for Cancer Research 19:43714382 